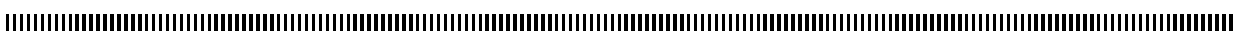

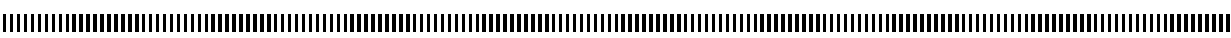

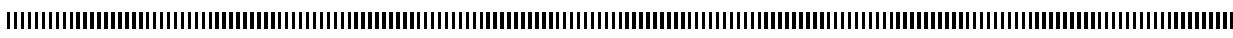

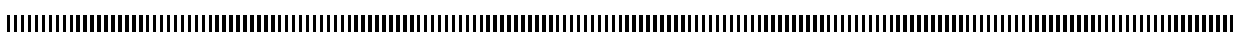

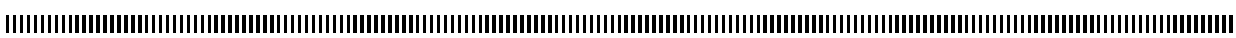
| |

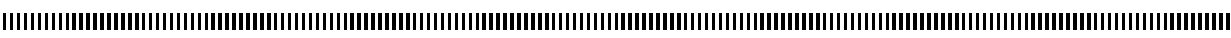

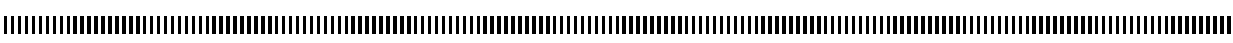
|

\title{
Survey of recent results of multi-compartments intra-host models of malaria and HIV
}

\author{
Samuel Bowong ${ }^{\mathrm{a}}$ — Jean Luc Dimi ${ }^{\mathrm{b}}$ — Jean Claude Kamgang ${ }^{\mathrm{c}}$ — Joseph \\ Mbang d _ Jean Jules Tewa e, \\ a Department of Mathematics and Computer Science, Faculty of Science, \\ University of Douala, P.O. Box 24157 Douala, Cameroon, \\ sbowong@gmail.com; samuelbowong@yahoo.fr \\ $\mathrm{b}$ Department of mathematics, Faculty of science, \\ University Marien Ngouabi of Brazzaville (Congo) \\ jldimi@yahoo.fr \\ c Department of Mathematics \\ ENSAI, University of Ngaoundere, Cameroon \\ jckamgang@gmail.com; jckamgang@yahoo.fr \\ d Department of Mathematics, Faculty of Science, \\ University of Yaoundé I, P.O. Box 812 Yaoundé, Cameroon \\ mbangjoh@yahoo.fr \\ e National Advanced School of Engineering \\ University of Yaounde I, Department of Mathematics and Physics \\ P.O. Box 8390 Yaounde, Cameroon \\ * Corresponding author \\ Tel.+(237) 77-71-13-69, Fax. +(237) 22-22-45-47 \\ tewajules@gmail.com; tewajules@yahoo.fr
}

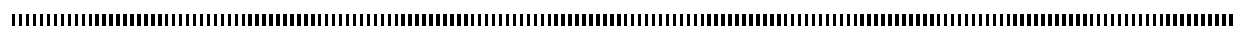

ABSTRACT. We present the recent results obtained for the within-host models of malaria and HIV. We briefly recall the Anderson-May-Gupter model. We also recall the Van Den Driessche method of computation for the basic reproduction ratio $\mathcal{R}_{0}$; here, a very simple formula is given for a new class of models. The global analysis of these models can be founded in [1, 2, 3, 5]. The results we recall here are for a model of one strain of parasites and many classes of age, a general model of $n$ strains of parasites and $k$ classes of age, a $S E_{1} E_{2} \cdots E_{n} I S$ model with one linear chain of compartments and finally a general $S E_{i 1} E_{i 2} \cdots E_{i n} I S$ model with $k$ linear chains of compartments. When $\mathcal{R}_{0} \leq 1$, the authors prove that there is a trivial equilibria calling disease free equilibrium (DFE) which is globally asymptotically stable (GAS) on the non-negative orthant, and when $\mathcal{R}_{0}>1$, they prove the existence of a unique endemic equilibrium in the non-negative orthant and give an explicit formula. They provided a weak condition for the global stability of endemic equilibrium.

RÉSUMÉ. Le travail que nous présentons ici est un résumé de quelques résultats récents obtenus dans $[1,2,3,5]$ concernant les modèles intra-hôtes multi-compartimentaux. II s'agit d'une analyse mathématique et globale des modèles intra-hôtes de paludisme et de V.I.H . Mais avant de présenter ces résultats, nous rappelons d'abord la méthode de calcul développée par Van Den Driessche[71] concernant le taux de reproduction de base $\mathcal{R}_{0}$ car c'est cette méthode qu'utilisent les auteurs dans 


\begin{abstract}
comme précurseur. Une formule simple est donnée ici pour le calcul de $\mathcal{R}_{0}$ dans les modèles étudiés. Les résultats que nous rappelons ici sont obtenus pour un modèle du paludisme à un génotype de parasites et $k$ classes d'âge, le modèle général à $n$ génotypes de parasites et $k$ classes d'âge, un modèle $S E_{1} E_{2} \cdots E_{n} I S$ avec une chaîne linéaire de parasites et enfin le modèle général $S E_{i 1} E_{i 2} \cdots E_{i n} I S$ avec $k$ chaîne linéaire de parasites. Lorsque $\mathcal{R}_{0} \leq 1$, les auteurs montrent qu'il existe un point d'équilibre évident, le DFE (Disease Free Equilibrium) qui est GAS (globalement asymptotiquement stable) sur l'orthant positif. Lorsque $\mathcal{R}_{0}>1$, ils montrent l'existence d'un unique équilibre endémique dans lórthant positif et moyennant une petite condition ils montrent que cet équilibre est globalement asymptotiquement stable.
\end{abstract}

KEYWORDS : Nonlinear dynamical systems, asymptotic stability, epidemic models, global stability.

MOTS-CLÉS : Systèmes dynamiques non linéaires, stabilité asymptotique, modèles épidémiologiques, stabilité globale.

| 


\section{Introduction on intrahost models of malaria and H.I.V}

Intra-host models of malaria describe the dynamics of the blood-stages of the parasites and their interaction with host-cells, in particular red blood cells (RBC) and immune effectors. Such models are used for different purposes : explain observations, predict impact of interventions, e.g. use of antimalarial drugs, estimate hidden states or parameters.

We give a brief review of the features of malaria. Malaria in a human begins with an inoculum of Plasmodium parasites (sporozoites) from a female Anophele mosquito. The sporozoites enter the liver within minutes. After a period of asexual reproduction in the liver, the parasites (merozoites) are released in the bloodstream where the asexual erythrocyte cycle begins. The merozoites enter red blood cells (RBC), grow and reproduce over a period of approximatively 48 hours after which the erythrocyte rupture releasing daughter parasites that quickly invade a fresh erythrocyte to renew the cycle. This blood cycle can be repeated many times, in the course of which some of the merozoites instead develop in the sexual form of the parasites : gametocytes. Gametocytes are benign for the host and are waiting for the mosquitoes.

In epidemiological models, we usually have two equilibrium points: an evident equilibrium calling Disease Free Equilibrium (DFE), which is the equilibrium point when there is no disease and the Endemic Equilibrium (EE). When the basic reproduction ratio $\mathcal{R}_{0} \leq 1$, the DFE is the only equilibrium in the non-negative orthant. We can show in this case that the DFE is globally asymptotically stable. When $\mathcal{R}_{0} \gg 1$, the other equilibrium is in the non-negative orthant and then is calling endemic equilibrium. Some authors have shown with minor condition that in this case the endemic equilibrium is globally asymptotically stable.

The evolution of the global analysis of multicompartmental models is not so easy. The fondamental paper is the paper of Lajmanovich and Yorke in 1976 on gonnorea, for $n$ groups. It's an $(S I S)^{n}$ model where the polutation of every sup-group is constant. The DFE here is the origin. Their result can be summarized as follows : if $\mathcal{R}_{0} \leq 1$ then there exists a unique equilibrium in the non-negative orthant, the DFE, which is globally asymptoticaly stable; if $\mathcal{R}_{0} \gg 1$ then there exists a unique endemic equilibrium in the non-negative orthant which is globally asymptoticaly stable on the model domain, except on the stable manifold of the DFE.

The global stability results of SEIR and SEIS models was conjectured for a long time. $\mathrm{Li}$ and Muldowney finally obtained it in 1985 . The proof uses the competitive nature of the system and Poincare-Bendixson properties in 3-dimension. In 2004, Korobeinikov obtained again Li and Muldowney results by using a Lyapounov function. In epidemiology, this kind of function was already used in 1986 by Beretta and Capasso, Lin and So in 1993 for partial results on global stability.

The first compartmental model was Ross-model in 1911. This original model has been extended in different directions. The first mathematical model of the erythrocyte cycle was proposed by Anderson, May and Gupta [7]. Like Ross-model, this original model has been also extended in different directions [6, 7, 25, 29, 32, 34, 65]. 
The original model of Anderson, May and Gupta [7] is given by the following system

$$
\left\{\begin{array}{l}
\dot{x}=\Lambda-\mu_{x} x-\beta x m \\
\dot{y}=\beta x m-\mu_{y} y \\
\dot{m}=r \mu_{y} y-\mu_{m} m-\beta x m
\end{array}\right.
$$

The state variables are denoted by $x, y$ and $m$. The variable $x$ denotes the concentration of uninfected red blood cells (RBC), $y$ the concentration of parasitized red blood cells (PRBC), $m$ the concentration of the free merozoites in the blood. The parameters $\mu_{x}$, $\mu_{y}$ and $\mu_{m}$ are the death rates of the RBC, PRBC and free merozoites respectively. The parameter $\beta$ is the contact rate between RBC and merozoites. Uninfected blood cells are recruited at a constant rate $\Lambda$ from the bone marrow and have a natural life-expectation of $1 / \mu_{x} \times$ days. Death of a PRBC results in the release of an average number of $r$ merozoites. Free merozoites die or successfully invade a RBC. A measurement of Plasmodium falciparum parasitaemia taken from a blood smear therefore samples young parasites only. Physician treating malaria use the number of parasites in peripheral blood smears as a measure of infection, but this does not gives the total parasites burden of the patient. In some respects this is a weak point of the model (1). Moreover antimalarial drugs are known to act preferentially on different stages of parasite development. This facts lead some authors to give a general approach to model the age structure of Plasmodium parasites $[26,27,28]$. The model is a catenary compartmental model. If we distinguish $k$ stages, the linear model is given by

$$
\left\{\begin{array}{l}
\dot{y}_{1}=r \gamma_{k}-\left(\mu_{1}+\gamma_{1}\right) y_{1} \\
\dot{y}_{2}=\gamma_{1} y_{1}-\left(\mu_{2}+\gamma_{2}\right) y_{2} \\
\cdots \\
\dot{y}_{i}=\gamma_{i-1} y_{i-1}-\left(\mu_{i}+\gamma_{i}\right) y_{i} \\
\cdots \\
\dot{y}_{k}=\gamma_{k-1} y_{k-1}-\left(\mu_{k}+\gamma_{k}\right) y_{k}
\end{array}\right.
$$

The state $y_{i}$ denotes the concentration in the blood of PRBC of class $i$. The rate transition from a compartment $i$ to the following $i+1$ is $\gamma_{i}$, the mortality is $\mu_{i}$. In the last $k$ stage the rupture of the erythrocyte releases $r$ merozoites, which invade, in this model, instantaneously fresh erythrocytes giving $r \gamma_{k}$ erythrocytes in stage 1.

This paper is organized as follows: In section 2, we describe the Van Den Driessche method of computation for the basic reproduction ratio $\mathcal{R}_{0}$. We apply it on a SIR model. In section 3, we present the results obtained by the authors of [1] where they analize an age-structured model for the dynamics of malaria infection which combines the advantages of two approches: the model presented is more general than the model of [26] since it considers different mortality and transmission factor for each stage. This model is based on a finite number of compartments, each representing a stage of development of the parasite inside the PRBC. The model describes the dynamics of the evolution of the morphological stages of the parasites and also makes allowance for the dynamics of the healthy RBC which is not the case in the model (2). In section 4, we present the results obtained by the authors of [1] where they generalized the model of two strains of parasites proposed by Hellriegel in [29] where she shows that the competition exclusion principle holds but only on simulations. They analyse a general model of $n$ strains of parasites and $k$ classes of age. In sections 5.1 and 5.2, we finally present the results obtained by the authors of [5] where they study a SEIS multicompartmental model of malaria. We have 
in this section a $S E_{1} E_{2} \cdots E_{n} I S$ model with 1 linear chain of compartments and the general $S E_{i 1} E_{i 2} \cdots E_{i n} I S$ model with $k$ linear chains of compartments.

\section{The Van Den Driessche method of computation for the basic reproduction ratio}

As usual, the basic reproduction number is the expected number of secondary cases produced in a completely susceptible population, by a typical infected individual during it's entire period of infectiousness [31, 20, 19].

The basic reproduction ratio can also be considerated as a bifurcation parameter. This parameter has a long history in epidemiology; his computation is not too easy. According to Van Den Driessche[71], the basic reproduction ratio of a given model can be defined as the spectral radius of the next generation matrix . The next generation matrix here is in the form $-F V^{-1}$, where $F$ is a non-negative matrix and $V$ a non-singular Metzler matrix. We try here to show how one can compute it by using the Van Den Driessche method. In order to apply this method, we use the following SIR model.

\subsection{A SIR model with two classes of age}

Let us consider the model given by the following figure

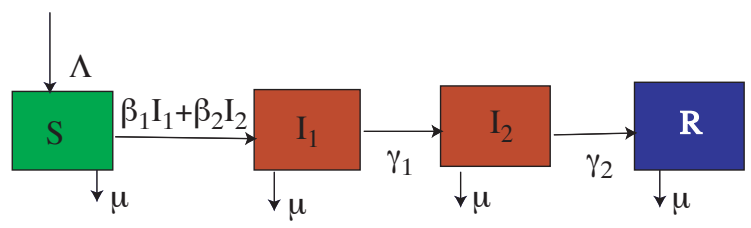

Figure 1. SIR model with 2 classes of age

The dynamics of the model is described by the following system:

$$
\left\{\begin{array}{l}
\dot{S}=\Lambda-\mu_{S} S-\left(\beta_{1} I_{1}+\beta_{2} I_{2}\right) S \\
\dot{I}_{1}=\left(\beta_{1} I_{1}+\beta_{2} I_{2}\right) S-\alpha_{1} I_{1} \\
\dot{I}_{2}=\gamma_{1} I_{1}-\alpha_{2} I_{2} \\
\dot{R}=\gamma_{2} I_{2}-\alpha_{3} R
\end{array}\right.
$$

where $\alpha_{1}=\mu+\gamma_{1}, \alpha_{2}=\mu+\gamma_{2}, \alpha_{3}=\mu$. This model contains $S E I R$ model when we suppose $\beta_{1}=0$. Except the last equation, the variable $R$ does not appears in the others; so, it has no influence on the dynamics of the other variables. We will then analize this model without equation $\dot{R}$.

We can also write our system in this form:

$$
\left\{\begin{array}{l}
\dot{S}=\Lambda-\mu_{S} S-S\langle\beta \mid I\rangle \\
\dot{I}=S\langle\beta \mid I\rangle \mathbf{b}+A I
\end{array}\right.
$$


where $\mathbf{b}=e_{1}=(1,0)^{T}, I=\left(I_{1}, I_{2}\right)^{T}, \beta=\left(\beta_{1}, \beta_{2}\right)^{T}$ and $A=\left[\begin{array}{cc}-\alpha_{1} & 0 \\ \gamma_{1} & -\alpha_{2}\end{array}\right]$.

For this model, the disease free equilibrium is $D F E=\left(S^{*}=\frac{\Lambda}{\mu}, 0\right)$. The non-negative orthant $\mathbb{R}_{+}^{3}$ is positively invariant. Let us compute the basic reproduction number of this model.

\subsection{The computation method of basic reproduction number}

We subdivide the population into 2 compartments; the susceptible $(\mathrm{S})$ individuals and the infectious (I) individuals. The number of individuals in the compartment $i$ is given by $x_{i}$ (or $x_{i}$ can also be an incidence, i.e. the percentage of individuals contained in the compartment $i$ ). We use the notation $x=(S, I)$, so that the first compartment is consisted of "no sick" individuals, precisely those who don't have germs (virus, protozoaire, parasites, ...).

In order to compute the basic reproduction number $\mathcal{R}_{0}$ like Van Den Driessche did[71], we consider the following figure:

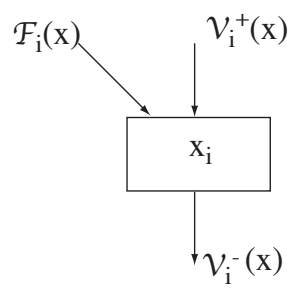

Figure 2. The dynamics of the compartment

The dynamics of the compartment $x_{i}$ is described by

$$
\dot{x}_{i}=\mathcal{F}(x)+\mathcal{V}^{+}(x)-\mathcal{V}^{-}(x)
$$

where $\mathcal{F}(x)$ denotes what is coming from the other compartments, due to the contamination.

$\mathcal{V}^{+}(x)$ denotes what is coming from the other compartments, due to another reasons. (deplacement, oldness, cureness etc ...)

$\mathcal{V}^{-}(x)$ denotes what disappear from the compartment.

We recall the definition of the spectral radius of a given matrix.

Definition 2.1 The spectral radius of a matrix $A$ is the maximum of it's eigenvalues modulus; ie

$$
\rho(A)=\max _{\lambda \in \operatorname{Sp}(A)}|\lambda|
$$


Theorem 2.2 :(Van Den Driessche)

For a given epidemiological model, if we have $D \mathcal{F}\left(S^{*}, 0\right)=\left(\begin{array}{cc}F & 0 \\ 0 & 0\end{array}\right)$ and $D \mathcal{V}\left(S^{*}, 0\right)=$ $\left(\begin{array}{cc}V & 0 \\ J_{1} & J_{2}\end{array}\right)$ where $F$ is a non-negative matrix and $V$ a Metzler-matrix, then the basic reproduction ratio $\mathcal{R}_{0}$ of the model is the spectral radius of the next generation matrix $M=-F V^{-1}$, ie

$$
\mathcal{R}_{0}=\rho\left(-F V^{-1}\right)
$$

\subsection{The computation of basic reproduction ratio for our SIR model}

For our SIR precedent model (3), we can observe that $S\langle\beta \mid I\rangle \mathbf{b}$ denotes what coming from the other compartments, due to the contamination. So, we have $\mathcal{F}(S, I)=(0, S\langle\beta|$ $I\rangle \mathbf{b})^{T}$

and $\mathcal{V}=(\Lambda-\mu S-\langle\beta \mid I\rangle S, A I)^{T}$. Their Jacobean matrices computed at DFE are

$$
\begin{gathered}
D \mathcal{F}\left(S^{*}, 0\right)=\left(\begin{array}{cc}
0 & 0 \\
0 & S^{*} \mathbf{b} \beta^{T}
\end{array}\right)=\left(\begin{array}{cc}
0 & 0 \\
0 & F
\end{array}\right) \\
D \mathcal{V}\left(S^{*}, 0\right)=\left(\begin{array}{cc}
-\mu & -S^{*} \beta^{T} \\
0 & A
\end{array}\right)=\left(\begin{array}{cc}
-\mu & -S^{*} \beta^{T} \\
0 & V
\end{array}\right)
\end{gathered}
$$

Then, according to the theorem 2.2, the basic reproduction ratio of the system (3) is

$$
\begin{aligned}
\mathcal{R}_{0} & =\rho\left(-F V^{-1}\right) \\
& =\rho\left(S^{*} \mathbf{b} \beta^{T}(-A)^{-1}\right)
\end{aligned}
$$

But $M=S^{*} \mathbf{b} \beta^{T}(-A)^{-1} \gg 0$ is a rank 1 matrix. We can then compute the basic reproduction number of the model as

$$
\begin{aligned}
\mathcal{R}_{0} & =S^{*} \beta^{T}(-A)^{-1} \mathbf{b} \\
& =S^{*}\left(\frac{\beta_{1}}{\alpha_{1}}+\frac{\beta_{2} \gamma_{1}}{\alpha_{1} \alpha_{2}}\right) \\
& =S^{*} \frac{\beta_{1} \alpha_{1}+\beta_{2} \gamma_{1}}{\alpha_{1} \alpha_{2}}
\end{aligned}
$$

Many authors use this method of computation, due to Van Den Driessche to obtain the basic reproduction ratio $\mathcal{R}_{0}$ of a given model. The results we have here are collecting in many papers of the epidemiological literature, especialy in $[1,2,3,5]$ where the authors use the Van Den Driessche method of computation of $\mathcal{R}_{0}$. We begin by an age-structured model for the dynamics of malaria infection.

\section{An age-structured model for the dynamics of malaria infection}

In many references cited, the recruitment of $\mathrm{RBC}$ is given by $\Lambda-\mu_{x} x$. The authors of $[1,2]$ used a more general function $\varphi(x)$. In a more complex system, the haemopoiesis 
could be an input coming from another system. They studied the following model with 1 strain of parasites and $k$ classes of age:

$$
\left\{\begin{array}{l}
\dot{x}=f(x)-\mu_{x} x-\beta x m=\varphi(x)-\beta x m \\
\dot{y}_{1}=\beta x m-\alpha_{1} y_{1} \\
\dot{y}_{2}=\gamma_{1} y_{1}-\alpha_{2} y_{2} \\
\cdots \\
\dot{y}_{k}=\gamma_{k-1} y_{k-1}-\alpha_{k} y_{k} \\
\dot{m}=r \gamma_{k} y_{k}-\mu_{m} m-u \beta x m
\end{array}\right.
$$

where $y$ denotes the column vector $\left(y_{1}, \cdots, y_{k}\right)^{T}$. The parameter $u$ only takes the value 0 or 1 . The reason of this parameter is to encompass some malaria models in which terms $-\beta x m$ or $\beta y m$ can appear or not. In [7], Anderson-May-Gupta have considered a term $-\beta x m$ in the equation $\dot{m}$. This corresponds to a loss of a free-merozoite once it enters a RBC. In [6], Anderson has considered a system without the $-\beta x m$ in the $\dot{m}$ equation. Then merozoites can infect RBC without themselves being absorbed, this allows one merozoite to infect more than one $R B C$. In [62], all the basic models of virus dynamics are also without this term. When $u=0$ the model can also be interpreted as a $S E_{1} \cdots E_{k} I$ epidemiological model. The gametocytes are benign and transmissible to mosquitoes. Like some authors in the epidemiological literature, they include a production of gametocytes in the model. If $y_{k+1}$ denotes the concentration of gametocytes the model becomes

$$
\left\{\begin{array}{l}
\dot{x}=f(x)-\mu_{x} x-\beta x m=\varphi(x)-\beta x m \\
\dot{y}_{1}=\beta x m-\alpha_{1} y_{1} \\
\dot{y}_{2}=\gamma_{1} y_{1}-\alpha_{2} y_{2} \\
\cdots \\
\dot{y}_{k}=\gamma_{k-1} y_{k-1}-\alpha_{k} y_{k} \\
\dot{y}_{k+1}=\delta \gamma_{k} y_{k}-\alpha_{k+1} y_{k+1} \\
\dot{m}=r \gamma_{k} y_{k}-\mu_{m} m-u \beta x m
\end{array}\right.
$$

The function $f$ gives the production of erythrocytes from the bone marrow. The function $\varphi(x)=f(x)-\mu_{x} x$ models the population dynamic of RBC in absence of parasites. The authors assumed that $f$ depends only of $x$ and that since homeostasis is maintained, the dynamic without parasites is asymptotically stable. In other words, for the system

$$
\dot{x}=f(x)-\mu_{x} x
$$

there exists a unique $x^{*}>0$ such that

$$
f\left(x^{*}\right)=\mu_{x} x^{*}, f(x)-\mu_{x} x>0 \text { for } 0 \leq x<x^{*}, f(x)-\mu_{x} x<0 \text { for } x>x^{*}
$$

The systems (4) and (5) can be written as

$$
\left\{\begin{array}{l}
\dot{x}=\varphi(x)-x\langle\beta \mid C z\rangle \\
\dot{z}=x\langle\beta \mid C z\rangle B+A z
\end{array}\right.
$$

where $x \in \mathbb{R}_{+}$denotes the class of susceptible erythrocytes, the components of $z \in$ $\mathbb{R}_{+}^{n}$ denotes the different class of latent erythrocytes, gametocytes and merozoites. The matrix $C$ is a nonzero $k \times n$ non-negative matrix, $\beta \in \mathbb{R}_{+}^{k}$ is a positive vector. The vector $B \in \mathbb{R}_{+}^{n}$ is a nonzero non-negative vector $(B>0)$ and $A$ is bounded by a stable Metzler 
matrix $\bar{A}, A \leq \bar{A}$. (A Metzler matrix is a matrix with off-diagonal entries non-negative $[9,36,52]$.) In this form this system encompasses numerous systems, particularly all the systems addressed by the authors in the paper [1].

The authors rewrite the system (4) as

$$
\left\{\begin{array}{l}
\dot{x}=f(x)-\mu_{x} x-\beta x m=\varphi(x)-\beta x m \\
\dot{y}=\beta x m e_{1}+A_{1} y \\
\dot{m}=r \gamma_{k} C y-\mu_{m} m-u \beta x m
\end{array}\right.
$$

where $e_{1}$ is the vector of $\mathbb{R}^{k}$ given by $e_{1}=(1,0, \cdots, 0)^{T}, C$ is the $k$ row matrix $C=$ $e_{k}^{T}=(0, \cdots, 0,1)$.

Their results are given in the following theorem

Theorem 3.1 Consider the system (4) or (5) with the hypothesis (6) satisfied. The basic reproduction ratio of the system (4) and (5) is given by

$$
\mathcal{R}_{0}=\frac{r \beta x^{*}}{\mu_{m}+u \beta x^{*}} \frac{\gamma_{1} \cdots \gamma_{k}}{\alpha_{1} \cdots \alpha_{k}}
$$

1) The non-negative orthant is positively invariant by the system (4).

2) Any trajectory of the system (4) is forward bounded.

3) The system (4) is globally asymptotically stable on $\mathbb{R}_{+}^{k+2}$ (respectively (5) on $\mathbb{R}_{+}^{k+3}$ ) at the disease free equilibrium (DFE) $\left(x^{*}, 0, \cdots, 0\right)$ if and only if $\mathcal{R}_{0} \leq 1$.

4) If $\mathcal{R}_{0}>1$ then the DFE is unstable and there exists a unique endemic equilibrium (EE) in the positive orthant, $(\bar{x}, \bar{y}, \bar{m}) \gg 0$ given by

$$
\left\{\begin{array}{l}
\bar{x}=\frac{\mu_{m}}{\beta\left[r \gamma_{k}\left(-A_{1}\right)^{-1}(k, 1)-u\right]}<x^{*}, \\
\bar{y}=\varphi(\bar{x})\left(-A_{1}\right)^{-1} e_{1}, \\
\bar{m}=\frac{f(\bar{x})-\mu_{x} \bar{x}}{\beta \bar{x}}=\frac{\varphi(\bar{x})}{\beta \bar{x}} .
\end{array}\right.
$$

Denoting $\alpha^{*}=-\max _{x \in\left[0, x^{*}\right]}\left(\varphi^{\prime}(x)\right)$, if

$$
u \beta \varphi(\bar{x}) \leq \alpha^{*} \mu_{m}
$$

then the endemic equilibrium is globally asymptotically stable on the non-negative orthant, excepted for initial conditions on the $x$-axis.

Proof: $\left(x B \beta^{T} C+\bar{A}\right)$ is a Metzler matrix if $x \geq 0$. So, if $z \geq 0$

$$
\dot{z}=\left(x B \beta^{T} C+A\right) z \leq\left(x B \beta^{T} C+\bar{A}\right) z
$$

With the hypothesis (6) $\varphi(0)>0$, the half line $\mathbb{R}_{+}$is positively invariant by $\dot{x}=\varphi(x)-$ $x\langle\beta \mid C z\rangle$. Since it is well known that linear Metzler systems let invariant the nonnegative orthant, this proves the positive invariance of the non-negative orthant $\mathbb{R}_{+}^{n+1}$ by (7). 
The expression of $\mathcal{R}_{0}$ is obtained easily by using the next generation matrix of the system (4) $[13,19,20]$. It can be observed that when the system is in the form (7), $x\langle\beta \mid C z\rangle B$ denotes what is coming from another compartments due to a contamination. It is straightforward using the definition of [20] to obtain

$$
\mathcal{R}_{0}=\left\langle\beta \mid C\left(-A^{-1}\right) B\right\rangle x^{*}
$$

For the global asymptotic stability of DFE, the authors considered the following candidate Lyapunov function:

$$
V=a_{2}\left(x-x^{*} \ln x\right)-a_{2} x^{*}\left(1-\ln x^{*}\right)+V_{D F E}(y, m)
$$

where $a_{2}=\frac{\alpha_{1} \cdots \alpha_{k}}{r \gamma_{1} \cdots \gamma_{k}} \frac{\mu_{m}}{\beta x^{*}}>0$ and $V_{D F E}$ is defined on the non-negative orthant by

$$
V_{D F E}(y, m)=y_{1}+\frac{\alpha_{1}}{\gamma_{1}} y_{2}+\cdots+\frac{\alpha_{1} \cdots \alpha_{k-1}}{\gamma_{1} \cdots \gamma_{k-1}} y_{k}+\frac{\alpha_{1} \cdots \alpha_{k}}{r \gamma_{1} \cdots \gamma_{k}} m
$$

This function is positive definite (relatively to $x^{*}$ ) on $\mathbb{R}_{+, x>0}^{k+2}=\left\{(x, y, m) \in \mathbb{R}_{+}^{k+2}\right.$ : $x>0\}$. Therefore,

$$
\dot{V}=\frac{a_{2}}{x}\left(x-x^{*}\right) \varphi(x)+\frac{\alpha_{1} \cdots \alpha_{k}}{r \gamma_{1} \cdots \gamma_{k}} \frac{\mu_{m}+u \beta x^{*}}{\beta x^{*}}\left(\mathcal{R}_{0}-1\right) \beta x m \leq 0
$$

By assumption (6) we have $\left(x-x^{*}\right) \varphi(x)=\left(x-x^{*}\right)\left(f(x)-\mu_{x} x\right) \leq 0$ for all $x \geq 0$. Therefore $\dot{V} \leq 0$ for all $(x, y, m) \in \mathbb{R}_{+, x>0}^{k+2}$ which proves the stability of the DFE. Its attractivity follows from the LaSalle's invariance principle [40] since the largest invariant set contained in $\left\{(x, y, m) \in \mathbb{R}_{+, x>0}^{k+2}: \dot{V}=0\right\}$ is reduced to the DFE. On the other hand the vector field is strictly entrant on the face $x=0$. Hence the whole orthant $\mathbb{R}_{+}^{k+2}$ belongs to the region of attraction of the DFE.

For the global asymptotic stability of the endemic equilibrium, the authors defined the following Lyapunov function on the non-negative orthant for the two systems corresponding to $u=0$ and $u=1$.

$$
V_{E E}(x, y, m)=a(x-\bar{x} \ln x)+\sum_{i=1}^{k} b_{i}\left(y_{i}-\bar{y}_{i} \ln y_{i}\right)+c(m-\bar{m} \ln m)
$$

where they choose the coefficients $a, b_{i}, c$ such that in the computation of $\dot{V}$ all the terms in $x m, y_{i}$ and $m$ cancel. Setting $\alpha^{*}=-\max _{x \in\left[0, x^{*}\right]} \varphi^{\prime}(x)$, they show that a sufficient condition for GAS of the EE is

$$
\mathcal{R}_{0}>1 \text { and } u \beta \varphi(\bar{x}) \leq \mu_{m} \alpha^{*}
$$

REMARK. - The authors define and compute the basic reproductive ratio $\mathcal{R}_{0}$ defined as in [19]. Equivalently a threshold condition can be defined related to the existence of a biological endemic equilibrium. To be more precise, the system (4) has always an equi$\operatorname{librium}(\bar{x}, \bar{y}, \bar{m})$ in $\mathbb{R}^{k+2}$.

REMARK. - It should be pointed out that the Lyapunov function defined by authors in [1] in order to show the global stability of the endemic equilibrium has a long history of application to Lotka-Volterra models $[22,23]$ and was originally discovered by Volterra 
himself, although he did not use the vocabulary and the theory of Lyapunov functions. Since epidemic models are "Lotka-Volterra" like models, the pertinence of this function is not surprising.

\section{Comparison with known results}

The stability result obtained here by the authors improves the one of De Leenher and Smith [17] in two directions:

1) it introduce $n$ stages for latent classes;

2) the sufficient condition (14) for the global asymptotic stability of the endemic equilibrium is weaker than the one provided in [17], for instance the sufficient condition given in theorem 3.1 is satisfied for the malaria parameters given in [7] while the condition of [17] is not satisfied.

Let us consider now the model studied in [1], which generalize the model studied in section 3.

\section{The general case : $n$ strains with $k$ classes of latent erythrocytes}

The authors of [1] analized the general case with $n$ strains of parasites and $k$ classes of latent erythrocytes.

They generalized easily the case of two strains with one class of parasitized RBC presented by Hellriegel in [29] where she showed that the competitive exclusion principle holds . The authors of [2] provide a mathematical proof of the simulation results obtained in [29] . It's important to remark that the system is no more competitive as soon as $k>1$. The model is defined by the following system with $k$ classes and $n$ strains

$$
\left\{\begin{array}{l}
\dot{x}=f(x)-\mu_{x} x-x \sum_{i=1}^{n} \beta_{i} m_{i}=\varphi(x)-x \sum_{i=1}^{n} \beta_{i} m_{i} \\
\text { for } i=1, \cdots, n \\
\dot{y}_{1, i}=\beta_{i} x m_{i}-\alpha_{1 i} y_{1, i} \\
\dot{y}_{2, i}=\gamma_{1, i} y_{1, i}-\alpha_{2, i} y_{2, i} \\
\cdots \\
\dot{y}_{k, i}=\gamma_{k-1, i} y_{k-1, i}-\alpha_{k, i} y_{k, i} \\
\dot{m}_{i}=r_{i} \gamma_{k, i} y_{k, i}-\mu_{m_{i}} m_{i}-u \beta_{i} x m_{i} \\
\dot{g}_{i}=\delta_{i} y_{k, i}-\mu_{g_{i}} g_{i}
\end{array}\right.
$$

The $u$ parameter is to encompass the case of $S E_{1} \cdots E_{k} I$ with $n$ strains.

The principal theorem of this paragraph is given by

Theorem 4.1 We consider the system (15) with the hypothesis (6) satisfied. We denote $\varphi(x)=f(x)-\mu_{x} x$.

The basic reproduction ratio $\mathcal{R}_{0}$ of the system (15) is given by

$$
\mathcal{R}_{0}^{i}=\frac{r_{i} \beta_{i} x^{*}}{\mu_{m_{i}}+u \beta_{i} x^{*}} \frac{\gamma_{1, i} \cdots \gamma_{k, i}}{\alpha_{1, i} \cdots \alpha_{k, i}}
$$

and

$$
\mathcal{R}_{0}=\max _{i=1, \cdots, n} \mathcal{R}_{0}^{i}
$$


1) The nonegative orthant is positively invariant by the system (15).

2) Any trajectory of the system (15) is forward bounded.

3) The system (15) is globally asymptotically stable on $\mathbb{R}_{+}$at the disease free equilibrium $(D F E)\left(x^{*}, 0, \cdots, 0\right)$ if and only if $\mathcal{R}_{0} \leq 1$.

4) If $\mathcal{R}_{0}>1$ then the DFE is unstable. If $R_{0}^{i}>1$ there exists an endemic equilibrium (EE) in the positive orthant corresponding to the genotype $i$, the value for the other indexes $j \neq i$ are $y_{j}=m_{j}=0$ and

$$
\left\{\begin{array}{l}
\bar{x}_{i}=\frac{\mu_{m_{i}}}{\beta_{i}\left[r_{i} \frac{\gamma_{1, i} \cdots \gamma_{k, i}}{\alpha_{1, i} \cdots \alpha_{k, i}}-u\right]} \\
\bar{y}_{i}=\varphi\left(\bar{x}_{i}\right)\left(-A_{i}\right)^{-1} e_{1} \\
\bar{m}_{i}=\frac{f\left(\bar{x}_{i}\right)-\mu_{x} \bar{x}_{i}}{\beta_{i} \bar{x}_{i}}=\frac{\varphi\left(\bar{x}_{i}\right)}{\beta_{i} \bar{x}_{i}} \\
\bar{g}_{i}=\frac{\delta_{i}}{\mu_{g_{i}}} \bar{y}_{k, i}
\end{array}\right.
$$

where the matrix $A_{i}$ is the analogous of the matrix $A_{1}$, defined precedently in section 3 , for the genotype $i$.

5) Assume $\mathcal{R}_{0}>1$ and define $\mathcal{T}_{0}^{i}=\frac{x^{*}}{\bar{x}_{i}}$,

Assume that the generic conditions $\mathcal{T}_{0}^{i} \neq \mathcal{T}_{0}^{j}$ are satisfied for $i \neq j$. We suppose that the genotypes have been indexed such that

$$
\mathcal{T}_{0}^{1}>\mathcal{T}_{0}^{2}>\cdots>\mathcal{T}_{0}^{n}
$$

Then the endemic equilibrium corresponding to $\bar{x}_{1}$ is asymptotically stable and the endemic equilibria corresponding to $\bar{x}_{j}$ for $j \neq 1$ (for those which are in the nonnegative orthant) are unstable.

6) We assume that the preceding hypothesis $\mathcal{T}_{0}^{1}>\mathcal{T}_{0}^{j}$ is satisfied with $\mathcal{R}_{0}>1$. We denote by $\alpha^{*}=-\max _{x \in\left[0, x^{*}\right]}\left(\varphi^{\prime}(x)\right)$. Then if

$$
\beta_{1} \varphi\left(\bar{x}_{1}\right) \leq \mu_{m_{1}} \alpha^{*}
$$

The equilibrium $\left(\bar{x}_{1}, \bar{y}_{1}, \bar{m}_{1}, \bar{g}_{1}, 0, \cdots, 0\right)$ is globally asymptotically stable on the nonnegative orthant minus the $x$-axis and the faces of the orthant defined by $y_{1}=m_{1}=$ $g_{1}=0$. In other words the most virulent strain 1 is the winner and the other strains goes extinct.

Proof: The system can be put in the form (7). So the basic reproduction ration is obtained like in section 2 by using the Van Den Driessche method of computation. The authors use the Lyapunov function

$$
V(y, m)=\sum_{i=1}^{n} V_{D F E}\left(y_{i}, m_{i}\right)
$$

where $V_{D F E}$ is defined by (12) to show the global stability of the DFE. For the global asymptotic stability of the endemic equilibrium, they had a similar condition as the condition (14) obtained in section 3.

REMARK. - On the non generic case it can be shown, using Lyapunov functions like the authors of [2] did, that there exists a continuum of stable endemic equilibria. Their result compares with the result of [11]. 
In the generic case, the dynamics of the system are completely determined. The nonnegative orthant is stratified in the union of stable manifolds corresponding to the different equilibria. Only the equilibrium corresponding to the winner strain has a basin of attraction with a non empty interior.

Let us consider now the $S E_{1} \cdots E_{n} I S$ and $S E_{i 1} \cdots E_{i n} I S$ models studied by the authors of [5].

\section{The $S E I S$ models with one and $k$ linear chain of compartments}

\subsection{The $S E_{1} \cdots E_{n} I S$ model with one linear chain of compartments}

The authors of [5] assume homogeneity of the susceptible population and neglect variations in susceptibility associated with disease spread. The population considered is divided into disjoint classes of susceptible, exposed, infective and recovered individuals, with numbers at time $t$ denoted by $S(t), E(t), I(t), R(t)$, respectively. All recruitements are within the susceptible class, and occurs at a constant rate $\Lambda$. On adequate contact with an infective, a susceptible individual becomes infected but is not yet infective. This individual remains in the exposed class for a certain latent period, passing through $n$ stages $\left(E_{1}, \cdots, E_{n}\right)$ before becoming infective. Once infective, an individual may either die due to the disease or, after an infective period, pass into the recovered class. For diseases that confer temporary immunity the individual returns to the susceptible class after an immune period. 
The transfer diagram for the system $S E_{1} \cdots E_{n} I S$ is given in the following figure

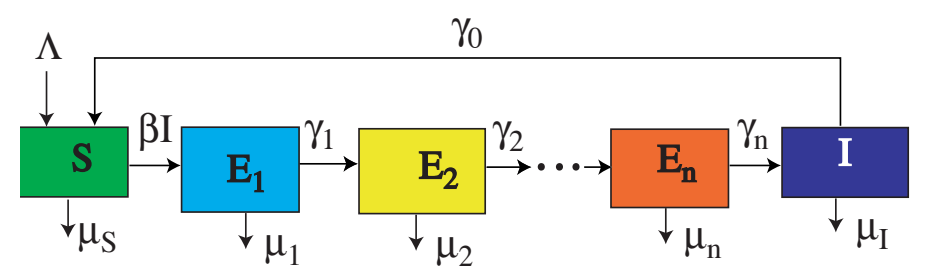

Figure 3. SEIS model

the dynamics of the model is described by the following system:

$$
\left\{\begin{array}{l}
\dot{S}=\Lambda-\mu_{S} S-\beta S I+\gamma_{0} I \\
\dot{E}_{1}=\beta S I-\alpha_{1} E_{1} \\
\dot{E}_{2}=\gamma_{1} E_{1}-\alpha_{2} E_{2} \\
\cdots \\
\dot{E}_{i}=\gamma_{i-1} E_{i-1}-\alpha_{i} E_{i} \\
\cdots \\
\dot{E}_{n}=\gamma_{n-1} E_{n-1}-\alpha_{n} E_{n} \\
\dot{I}=\gamma_{n} E_{n}-\alpha_{n+1} I
\end{array}\right.
$$

Setting $x=S$ and $y_{i}=E_{i}$ for $i=1, \cdots, n$ and $y_{n+1}=I$, the system (17) can be written in the form :

$$
\left\{\begin{array}{l}
\dot{x}=\varphi(x)-\beta x\left\langle y \mid e_{\omega}\right\rangle+\gamma_{0} y_{n+1} \\
\dot{y}=\beta x\left\langle y \mid e_{\omega}\right\rangle e_{1}+A y
\end{array}\right.
$$

where $e_{1}=(1,0,0, \ldots, 0)^{T}, e_{\omega}=(0,0, \ldots, 0,1)^{T}, \varphi(x)=\Lambda-\mu_{x} x$ and $\langle\mid\rangle$ denotes the usual scalar product. The matrix $A$ is given by 


$$
A=\left(\begin{array}{cccccc}
-\alpha_{1} & 0 & 0 & \ldots & 0 & 0 \\
\gamma_{1} & -\alpha_{2} & 0 & \ldots & 0 & 0 \\
0 & \gamma_{2} & -\alpha_{3} & \ldots & 0 & 0 \\
\vdots & \ddots & \ddots & \ddots & \vdots & \vdots \\
0 & 0 & \ldots & \gamma_{n-1} & -\alpha_{n} & 0 \\
0 & 0 & \ldots & 0 & \gamma_{n} & -\alpha_{n+1}
\end{array}\right)
$$

The principal results obtained by the authors are in the following theorem

Theorem $5.1 \quad$ 1) For $\epsilon \geq 0$, the simplex:

$$
\Omega_{\epsilon}=\left\{\left(S, E_{1}, \cdots, E_{n}, I\right) \in \mathbb{R}_{+}^{n+2} \mid N(t) \leq \frac{\Lambda}{\mu}+\epsilon\right\}
$$

is a compact invariant set for the system (17) and for $\epsilon>0$, this set is absorbant. So, the analize of authors is limited to this simplex for $\epsilon>0$. The basic reproduction number is given by:

$$
\mathcal{R}_{0}=\beta x^{*}\left\langle e_{\omega} \mid-A^{-1} e_{1}\right\rangle=\beta x^{*} \frac{\gamma_{1} \ldots \gamma_{n}}{\alpha_{1} \ldots \alpha_{n}} \frac{1}{\alpha_{n+1}}
$$

2) For the system (17), when $\mathcal{R}_{0} \leq 1$, the DFE is globally asymptoticaly stable on $\Omega_{\epsilon}$; this implies the global stability in the non-negative orthant $\mathbb{R}_{+}^{n+3}$. The disease dies out and all the parasites disappear.

3) When $\mathcal{R}_{0}>1$, there exists a unique strong endemic equilibrium which is globally asymptoticaly stable on the positive orthant except the positive half $x$-axis, which is the stable manifold of the DFE.

Proof: For the computation of the basic reproduction ratio $\mathcal{R}_{0}$, we can recognize the expression $\beta x\left\langle y \mid e_{\omega}\right\rangle e_{1}$ in the system (18) which coming from another compartments due to contamination. The Van Den Driessche method in section 2 can be used. For the global stability of the DFE, the authors used the following Lyapunov-LaSalle function:

$$
V_{D F E}=\beta\left\langle(-A)^{-T} e_{\omega} \mid y\right\rangle
$$

The endemic equilibrium $(\bar{x}, \bar{y})$ of ( 17) satisfies these equations:

$$
\bar{x}=\frac{x^{*}}{\mathcal{R}_{0}}>0
$$

Since $\mathcal{R}_{0}>1$, we have $\bar{x}<x^{*}$, and (6) shows that $\varphi(\bar{x})>0$. Or

$$
\beta \bar{x}=\frac{\alpha_{1} \ldots \alpha_{n}}{\gamma_{1} \ldots \gamma_{n}} \alpha_{n+1}>\alpha_{n+1}>\gamma_{0}
$$

So,

$$
\bar{y}_{n+1}=\frac{\varphi(\bar{x})}{\beta \bar{x}-\gamma_{0}}>0
$$

and 


$$
\bar{y}=\frac{\beta \bar{x}}{\beta \bar{x}-\gamma_{0}} \varphi(\bar{x})\left(-A^{-1}\right) e_{1}
$$

For the proof of the global asymptotic stability of the endemic equilibrium, the authors of [5] consider as in section 4 the following function $V_{E E}$, defined on the non-negative orthant $\mathbb{R}_{+}^{n+2}$ :

$$
V_{E E}=a_{0}(x-\bar{x} \ln x)+\sum_{i=1}^{n+1} a_{i}\left(y_{i}-\bar{y}_{i} \ln y_{i}\right)-K
$$

with $K=a_{0}(\bar{x}-\bar{x} \ln \bar{x})+\sum_{i=1}^{n+1} a_{i}\left(\bar{y}_{i}-\bar{y}_{i} \ln \bar{y}_{i}\right)$ where they choose the coefficients $\left(a_{0}, a\right) \in \mathbb{R}_{+}^{n+2}$ so that in the expression of $\dot{V}_{E E}$ along the trajectories of (17), all the linear terms in $x, y_{1}, \ldots, y_{n}$ and bilinear terms in $x y_{n+1}$ disappear.

It's derivative is given by:

$$
\begin{aligned}
\dot{V}_{E E}= & -\mu \frac{(x-\bar{x})^{2}}{x}+\gamma_{0}\left(y_{n+1}-\bar{y}_{n+1}\right) \frac{(x-\bar{x})}{x} \\
& +\beta \bar{x} \bar{y}_{n+1}\left[(n+2)-\frac{\bar{x}}{x}-\frac{x}{\bar{x}} \frac{y_{n+1}}{\bar{y}_{n+1}} \frac{\bar{y}_{1}}{y_{1}}-\frac{y_{1}}{\bar{y}_{1}} \frac{\bar{y}_{2}}{y_{2}}-\cdots-\frac{y_{n-1}}{\bar{y}_{n-1}} \frac{\bar{y}_{n}}{y_{n}}-\frac{y_{n}}{\bar{y}_{n}} \frac{\bar{y}_{n+1}}{y_{n+1}}\right]
\end{aligned}
$$

They used the inequality between the arithmetical and geometrical means to deduce that, excepted the term $\gamma_{0}\left(y_{n+1}-\bar{y}_{n+1}\right) \frac{(x-\bar{x})}{x}$ the two others are negative. Using the relation

$$
\dot{x}=-\left(\mu+\beta y_{n+1}\right)(x-\bar{x})-\left(y_{n+1}-\bar{y}_{n+1}\right)\left(\beta \bar{x}-\gamma_{0}\right)
$$

they consider the following candidate Lyapunov function

$$
V(x, y)=\frac{\gamma_{0}}{\beta \bar{x}-\gamma_{0}}(x-\bar{x} \ln x)+V_{E E}
$$

The preceding computation gives

$$
\begin{aligned}
\dot{V}= & -\mu \frac{(x-\bar{x})^{2}}{x}-\left(\mu+\beta y_{n+1}\right) \frac{(x-\bar{x})^{2}}{x} \\
& +\beta \bar{x} \bar{y}_{n+1}\left[(n+2)-\frac{\bar{x}}{x}-\frac{x}{\bar{x}} \frac{y_{n+1}}{\bar{y}_{n+1}} \frac{\bar{y}_{1}}{y_{1}}-\frac{y_{1}}{\bar{y}_{1}} \frac{\bar{y}_{2}}{y_{2}}-\cdots-\frac{y_{n-1}}{\bar{y}_{n-1}} \frac{\bar{y}_{n}}{y_{n}}-\frac{y_{n}}{\bar{y}_{n}} \frac{\bar{y}_{n+1}}{y_{n+1}}\right]
\end{aligned}
$$

This proves that $\dot{V}$ is negative definite on the non-negative orthant. 


\subsection{The $S E_{i 1} \cdots E_{i n} I S$ models with k linear chain of compartments}

In order to model a distribution lag which is approximated by a convex sum of Erlang distribution (probability density function), the authors in [5] consider a model with $k$ chains.

They wrote the system in a analogous form as (18) with some modifications where $y$ denotes the state vector corresponding to the latent classes and decomposed the vector $y$ in $k$ blocks, $y=\left(y_{1}, y_{2}, \ldots, y_{k}\right)$ corresponding to the $k$ parallel chains. Each vector-block $y_{i}$ is of length $n_{i}$, the length of the $i$ th-chain. $y_{i}=\left(y_{i, 1}, \cdots, y_{i, \omega}\right)$ with the convention that $y_{i, \omega}$ is the last component of $y_{i}$. The notation $\omega$ plays a role similar to the syntax end in MATLAB. We denote by $x$ and $z$ the state of the susceptible and infectious respectively. The state vector $y$ is in $\mathbb{R}^{n}$, with $n=\sum n_{i}$, and the system is in $\mathbb{R}^{n+2}$. As usual, by misuse of language, they identify $\mathbb{R}^{n_{i}}$ with it's canonical injection in $\mathbb{R}^{n+2}$. According to this identification, $e_{i, j}$ will denote the $j$-th canonical vector of $\mathbb{R}^{n_{i}}$, in other words it is the vector numbered $1+n_{1}+\cdots+n_{i-1}+j$. The last vector of $\mathbb{R}^{n_{i}}$ will be denoted by $e_{i, \omega}$ and the last vector of $\mathbb{R}^{n+2}$, corresponding to $z$, will be denoted by $e_{\omega}$.

The figure is given by

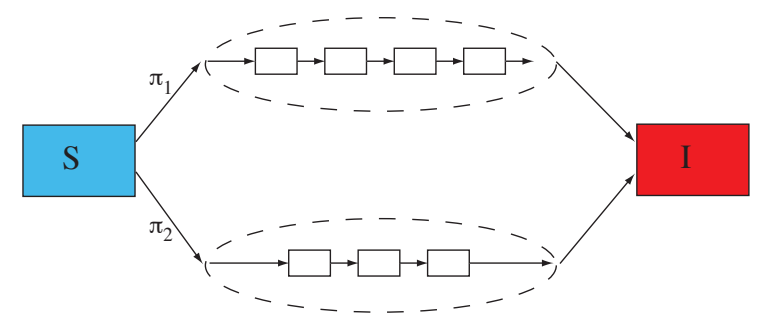

Figure 4. The $S E_{1} \cdots E_{n} I S$ model with $k$ linear chain of compartments

Then the dynamics of the model is given by the following system

$$
\left\{\begin{aligned}
\dot{x} & =\varphi(x)-\beta x z+\gamma_{0} z \\
\dot{y} & =\beta x z \mathbf{b}+A y \\
\dot{z} & =\mathbf{c}^{T} y-\alpha_{z} z
\end{aligned}\right.
$$

where $A$ is a $n \times n$ diagonal block matrix $A=\operatorname{diag}\left(A_{1}, \cdots, A_{k}\right)$. The $i$ block $A_{i}$ is a $n_{i} \times n_{i}$ matrix as in (5.1), where the coefficients $\alpha$ and $\beta$ appearing in (5.1) are indexed by $i$, i.e $\alpha_{i, j}$ for $j=1, \cdots, n_{i}$ and $\gamma_{i, j}$ for $j=1, \cdots, j-1$.

The matrix $\mathbf{c}^{T}$ is a $1 \times n$ matrix given by

$$
\mathbf{c}^{T}=\left[\gamma_{1, \omega} e_{1, \omega}^{T}, \cdots, \gamma_{k, \omega} e_{k, \omega}^{T}\right]
$$


corresponding to each output of the last compartment of each latent chain entering the infectious compartment.

The vector $\mathbf{b}$ is

$$
\mathbf{b}=\pi_{1} e_{1,1}+\cdots+\pi_{k, 1} e_{k, 1}
$$

corresponding to the input in each first compartment of each latent chain, with $\pi_{1}+\cdots+$ $\pi_{k}=1$.

Denoting by $\tilde{A}$ the block matrix

$$
\tilde{A}=\left[\begin{array}{cc}
A & 0 \\
\mathbf{c}^{T} & -\alpha_{z}
\end{array}\right]
$$

their principal results are given in this theorem

Theorem 5.2 For the system (20),

the basic reproduction ratio of the system is given by

$$
\mathcal{R}_{0}=\beta x^{*}\left\langle e_{\omega} \mid\left(-\tilde{A}^{-1}\right) \mathbf{b}\right\rangle
$$

1) The system (20) is globally asymptotically stable on $\mathbb{R}_{+}^{n+2}$ at the DFE $\left(x^{*}, 0, \ldots, 0\right)$ if and only if $\mathcal{R}_{0} \leq 1$.

2) If $\mathcal{R}_{0}>1$ then the DFE is unstable, there exists a unique strongly endemic equilibrium $(\bar{x}, \bar{y}, \bar{z}) \gg 0$ in the positive orthant. The endemic equilibrium is globally asymptotically stable on the non-negative orthant, excepted for initial conditions on the non-negative $x$-axis.

Proof: The system (20) is in the form (18) used in section 5.1. In that form, the computation of $\mathcal{R}_{0}$ is the same as in section 2. For the global stability of DFE when $\mathcal{R}_{0} \leq 1$, the authors used in [5] the following Lyapunov-LaSalle function :

$$
V_{D F E}=\beta\left\langle(-\tilde{A})^{-T} e_{\omega} \mid\left(\begin{array}{l}
y \\
z
\end{array}\right)\right\rangle
$$

When $\mathcal{R}_{0}>1$, the endemic equilibrium exists and we have

$$
\left\{\begin{aligned}
\bar{x} & =\frac{x^{*}}{\mathcal{R}_{0}} \\
\bar{y} & =\varphi(\bar{x})(-A)^{-1} \mathbf{b} \\
\bar{z} & =\frac{\varphi(\bar{x})}{\beta \bar{x}-\gamma_{0}}
\end{aligned}\right.
$$

For the global stability of endemic equilibrium when $\mathcal{R}_{0} \geq 1$, the authors used in [5] the following function

$$
V_{E E}=a_{0}(x-\bar{x} \ln x)+\sum_{i=1}^{k} a_{i}\left(y_{i}-\bar{y}_{i} \ln y_{i}\right)+a_{n+2}(z-\bar{z} \ln z)-K
$$


It's derivative gives

$$
\begin{aligned}
\dot{V}_{E E} & =a_{0} \varphi(x) \frac{(x-\bar{x})}{x}+\gamma_{0}(z-\bar{z}) \frac{(x-\bar{x})}{x} \\
& +\beta \bar{x} \bar{z} \sum_{i=1}^{k} \pi_{i} a_{i, 1}\left[\left(n_{i}+2\right)-\frac{\bar{x}}{x}-\frac{y_{i, 1}}{\bar{y}_{i, 1}} \frac{x}{\bar{x}} \frac{z}{\bar{z}}\right. \\
& \left.-\sum_{j=2}^{n_{i}} \frac{y_{i, j-1}}{\bar{y}_{i, j-1}} \frac{\bar{y}_{i, j}}{y_{i, j}}-\frac{y_{i, \omega}}{\bar{y}_{i, \omega}} \frac{\bar{z}}{z}\right]
\end{aligned}
$$

With this expression of $\dot{V}_{E E}$, we have like in the case of one linear chain in subsection 5.1 that

$$
V(x, y, z)=\frac{\gamma_{0}}{\beta \bar{x}-\gamma_{0}}(x-\bar{x} \ln x)+V_{E E}(x, y, z)
$$

is a Lyapunov strict function.

\section{Conclusion}

In section 2, we have presented the Van Den Driessche method of computaion for the basic reproduction ratio $\mathcal{R}_{0}$. This method is used in epidemiology literature by many authors, like the authors of $[1,2,3,5]$.

In sections 3 and 4, we have given the results obtained by the authors of [1,2], where they obtained the global asymptotic stability of the DFE and the following condition for the global asymptotic stability of the endemic equilibrium

$$
\mathcal{R}_{0} \gg 1 \text { and } u \beta \varphi(\bar{x}) \leq \mu_{m} \alpha^{*}
$$

The stability result obtained here by the authors improves the one of De Leenher and Smith [17].

In subsections 5.1 and 5.2, we remark that the authors have given a complete analysis of the models $S E_{1} \cdots E_{n} I R$ with latent classes. The latent classes are made up by parallel chains of different lengths and are inserted between the susceptible and the infectious compartments. They compute the basic reproduction ratio $\mathcal{R}_{0}$ as in section 2 and prove that if $\mathcal{R}_{0} \leq 1$, the disease free equilibrium is globally asymptotically stable on the non-negative orthant. If $\mathcal{R}_{0}>1$ then a unique endemic equilibrium exists in the non-negative orthant and is globally asymptotically stable without condition, on the non-negative orthant, excepted on the S-axis.

Acknowledgements: This work was supported by EpiMath network ${ }^{1}$.

Many thanks to Professor Claude Lobry for his contribution to African scientific building. Many thanks also to Professor Gauthier Sallet of INRIA LORRAINE and MetzUniversity (France) for his work in the African continent, and his assistance to EpiMath network. The authors will not forget Martin Eichner of Tubingen-University, Christophe Rogier of IMTSSA in Marseille (France) and A. Iggidr of INRIA LORRAINE and Metz-University (France) for their contribution and assistance to EpiMath network. 


\section{References}

[1] A. IgGidR, J.C. KAMgAng, G. SAlLEt, JJ. TEWA, Global analysis of new intrahost models with a competitive exclusion principle, SIAM J. APPL. MATH, Vol 67, $\mathrm{N}_{i} 1$, pp. 260-278; December 2006.

[2] P. Adda, J.L. Dimi, A. Iggidr, J.C. Kamgang, G. Sallet, JJ. Tewa,General models of host-parasite systems. Global analysis, Discrete and Continuous Dynamical Systems-Series B, Vol 8, Ni1, pp. 1-17; July 2007.

[3] A. Iggidr, J. Mbang, G. Sallet, J.J. Tewa ; Ç Multi-Compartment Models È ; AIMS Journal : Discrete and Continuous Dynamical Systems Đ Series B (DCDS-B), Supplement, 2007 ; pp. 506-519, september 2007

[4] A. FAll, A. IGgidr, G. SAllet, JJ. TEWA,Epidemiological models and Lyapunov functions; Mathematical Modelling of Natural Phenomena, Vol 2, $\mathrm{N}_{\mathrm{i}} 1$, pp.55-71; 2006

[5] N. BAme, S. Bowong, J. MBAng, G. SAllet, JJ. Tewa;Global stability Analysis for SEIS models with $n$ latent classes; Mathematical Biosciences and Engineering, Vol 5, $\mathrm{N}_{\mathrm{i}} 1$, pp.20-33, january 2008

[6] R. M. ANDERSON, Complex dynamic behaviours in the interaction between parasite population and the host's immune system., Int J Parasitol, 28 (1998), pp. 551-566.

[7] R. M. Anderson, R. M. May, And S. GuptA, Non-linear phenomena in host-parasite interactions., Parasitology, 99 Suppl (1989), pp. S59-79.

[8] A BERETTA AND V. CAPASSO, On the general structure of epidemic systems. global asymptotic stability, Comput. Math. Appl., (1986), pp. 677-694.

[9] A. Berman AND R. J. Plemmons, Nonnegative matrices in the mathematical sciences., SIAM, 1994.

[10] N. P. Bhatia And G. P. Szegö, Dynamical systems: Stability theory and applications, Springer-Verlag, 1967.

[11] H.J. BREMERMANN AND H. R. THIEME, A competitive exclusion principle for pathogen virulence, J. Math. Biol., (1989), pp. 179-190.

[12] G.J. BUtLeR, , H.S.B. HSU, AND P. WALTMAN, coexistence of competing predator in a chemostat, J. Math. Biol., (1983), pp. 133-151.

[13] C. Castillo-Chavez, Z. Feng, And W. Huang, On the computation of $R_{0}$ and its role on global stability, in Mathematical approaches for emerging and reemerging infectious diseases: an introduction (Minneapolis, MN, 1999), vol. 125 of IMA Vol. Math. Appl., Springer, New York, 2002, pp. 229-250.

[14] C. Castillo-Chavez, W. Huang, AND J. Li, Competitive exclusion in gonorrhea models and other sexually transmitted diseases, SIAM J. Appl. Math., 56 (1996), pp. 494-508.

[15] C. Castillo-Chavez, W. Huang, And J. Li, Competitive exclusion and coexistence of multiple strains in an SIS STD model, SIAM J. Appl. Math., 59 (1999), pp. 1790-1811.

[16] W. E. COLlins AND G. M. JEFFERY, A retrospective examination of the patterns of recrudescence in patients infected with plasmodium falciparum., Am J Trop Med Hyg, 61 (1999), pp. 44-48.

[17] P. De Leenheer And H. L. Smith, Virus dynamics: A global analysis., SiAM J. Appl. Math., 63 (2003), pp. 1313-1327.

[18] H. H. Diebner, M. Eichner, L. Molineaux, W. E. Collins, G. M. Jeffery, And K. DIETZ, Modelling the transition of asexual blood stages of Plasmodium falciparum to gametocytes., J. Theor. Biol., 202 (2000), pp. 113-127.

[19] O. Diekmann, J. A. P. Heesterbeek, And J. A. J. Metz, On the definition and the computation of the basic reproduction ratio $R_{0}$ in models for infectious diseases in heterogeneous 
populations, J. Math. Biol., 28 (1990), pp. 365-382.

[20] - Mathematical epidemiology of infectious diseases, Wiley Series in Mathematical and Computational Biology, John Wiley \& Sons Ltd., Chichester, 2000. Model building, analysis and interpretation.

[21] K. DiETZ, Epidemiologic interference of virus population, J. Math. Biol., 8 (1979), pp. 291 300.

[22] B. S. GoH, Global stability in two species interactions, J. Math. Biol., (1976), pp. 313-318.

[23] _ Global stability in many-species systems, Amer.Naturalist, (1977), pp. 135-143.

[24] M B Gravenor AND D KwiatKowski, An analysis of the temperature effects of fever on the intra-host population dynamics of plasmodium falciparum., Parasitology, 117 ( Pt 2) (1998), pp. 97-105.

[25] M. B. GRAVENOR AND A.L. LloYD, Reply to : Models for the in-host dynamics of malaria revisited : errors in some basic models lead to large over-estimates of growth rates, Parasitology, 117 (1998), pp. 409-410.

[26] M. B. Gravenor, A. L. Lloyd, P. G. Kremsner, M. A. Missinou, M. English, K. MARSH, AND D. KWIATKOWSKI, A model for estimating total parasite load in falciparum malaria patients., J Theor Biol, 217 (2002), pp. 137-48.

[27] M. B. Gravenor, A. R. MCLeAn, And D. KwiatKowski, The regulation of malaria parasitaemia: parameter estimates for a population model., Parasitology, 110 ( Pt 2) (1995), pp. 115-22.

[28] M. B. Gravenor, M. B. Van Hensbroek, and D. Kwiatkowski, Estimating sequestered parasite population dynamics in cerebral malaria., Proc Natl Acad Sci U S A, 95 (1998), pp. 7620-7624.

[29] B. HeLlRIEGEL, Modelling the immune response to malaria with ecological concepts: shortterm behaviour against long-term equilibrium., Proc R Soc Lond B Biol Sci, 250 (1992), pp. 249-256.

[30] H. W. Hethcote And H. R. Thieme, Stability of the endemic equilibrium in epidemic models with subpopulations, Math. Biosci., 75 (1985), pp. 205-227.

[31] — The mathematics of infectious diseases, SIAM Rev., 42 (2000), pp. 599-653 (electronic).

[32] C. Hetzel AND R. M. Anderson, The within-host cellular dynamics of bloodstage malaria: theoretical and experimental studies., Parasitology, 113 ( Pt 1) (1996), pp. 25-38.

[33] M.W. HIRSCH, The dynamical systems approach to differential equations, Bull A.M.S., (1984), pp. 1-64.

[34] M. B. Hoshen, R. Heinrich, W. D. Stein, And H. Ginsburg, Mathematical modelling of the within-host dynamics of Plasmodium falciparum., Parasitology, 121 ( Pt 3) (2001), pp. 227-235.

[35] J. A. JACQUEZ, Compartmental Analysis in Biology and Medicine, Biomedware, Ann Arbor MI, 1996.

[36] J. A. JACQUEZ AND C. P. SimOn, Qualitative theory of compartmental systems, SIAM Rev., 35 (1993), pp. 43-79.

[37] J. A. Jacquez, C. P. Simon, And J. Koopman, Core groups and the ROs for subgroups in heterogeneous sis and si models, in Epidemics models : their structure and relation to data, D.Mollison, ed., Cambridge University Press, 1996, pp. 279-301.

[38] A. Korobeinikov AND P.K MAINI, A lyapunov function and global properties for sir and seir epidemiolgical models with nonlinear incidence, Math Biosci and Engineering, (2004), pp. 57-60. 
[39] A. LAJMANOVICH AND J.A. YORKE, A deterministic model for gonorrhea in a nonhomogeneous population., Math. Biosci., 28 (1976), pp. 221-236.

[40] J.P. LASALLE AND S. LEFSCHETZ, Stability by Liapunov's direct method with applications, Academic Press, New-York, 1961.

[41] S.A. LEVIN AND D. PIMENTEL, Selection of intermediate rates increase in parasite-host systems, Amer.Naturalist, (1981), pp. 308-315.

[42] M.Y. LI, J. R. GRAEF, L. WANG, AND J. KARSAI, Global dynamics of a SEIR model with varying total population size, Math. Biosci., 160 (1999), pp. 191-213.

[43] M.Y. Li, J. S. Muldowney, AND P. VAN DEN DRIESSCHE, Global stability for the SEIR model in epidemiology, Math. Biosci., 125 (1995), pp. 155-164.

[44] - Global stability of SEIRS models in epidemiology, Canad. Appl. Math. Quart., 7 (1999), pp. 409-425.

[45] M. Y. Li AND J. S. MuldownEY, Global stability for the SEIR model in epidemiology, Math. Biosci., 125 (1995), pp. 155-164.

[46] - A geometric approach to global-stability problems, SIAM J. Math. Anal., 27 (1996), pp. 1070-1083.

[47] M. Y. Li, H. L. SMITH, AND L. WANG, Global dynamics an SEIR epidemic model with vertical transmission, SIAM J. Appl. Math., 62 (2001), pp. 58-69 (electronic).

[48] X. Lin AND J. W.-H. So, Global stability of the endemic equilibrium and uniform persistence in epidemic models with subpopulations., J. Aust. Math. Soc., Ser. B, 34 (1993), pp. 282 295.

[49] A. L. LlOYD, The dependance of viral parameter estimates on the assumed viral life cycle: limitations of studies of viral load data, Proc R Soc Lond B Biol Sci, (2001), pp. 847-854.

[50] - Destabilization of epidemic models with the inclusion of realistic distributions of infectious periods., Proc R Soc Lond B Biol Sci, 268 (2001), pp. 985-993.

[51] - Realistic distributions of infectious periods in epidemic models: changing patterns of persistence and dynamics., Theor Popul Biol, 60 (2001), pp. 59-71.

[52] D. G. Luenberger, Introduction to dynamic systems. Theory, models, and applications., John Wiley \& Sons Ltd., 1979.

[53] N. MacDonald, Time Lags in Biological Models, Springer-Verlag, 1978.

[54] D. P. Mason, F. E. MCKenZIE, And W. H. Bossert, The blood-stage dynamics of mixed plasmodium malariae-plasmodium falciparum infections., J Theor Biol, 198 (1999), pp. 549566.

[55] R. M. MAY AND R. M. ANDERSOn, Epidemiology and genetics in the coevolution of parasites and hosts., Proc R Soc Lond B Biol Sci, 219 (1983), pp. 281-313.

[56] J. Maynard Smith, Models in ecology, Cambridge University Press, 1974.

[57] F.E. MCKENZIE AND W.H. BOSSERT, The dynamics of plasmodium falciparum blood-stage infection, J.theor. Biol., 188 (1997), pp. 127-140.

[58] F. E. MCKENZIE AND W. H. BOSSERT, The optimal production of gametocytes by plasmodium falciparum., J Theor Biol, 193 (1998), pp. 419-428.

[59] P.G MCQUEEN AND F. E. MCKENZIE, Age-structured red blood cell susceptibility and the dynamics of malaria infections., Proc Natl Acad Sci U S A, 101 (2004), pp. 9161-9166.

[60] L. Molineaux, H. H. Diebner, M. Eichner, W. E. Collins, G. M. Jeffery, And K. DIETZ, Plasmodium falciparum parasitaemia described by a new mathematical model., Parasitology, 122 (2001), pp. 379-391.

[61] L. Molineaux And K. Dietz, Review of intra-host models of malaria., Parassitologia, 41 (2000), pp. 221-231. 
[62] M. A. NowaK AND R. M. MaY, virus dynamics. Mathematical principles of immunology and virology, Oxford University Press, 2000.

[63] A. S. Perelson, D. E. Kirschner, And R. De Boer, Dynamics of HIV infection of CD4+ T cells., Math Biosci, 114 (93), pp. 81-125.

[64] A. S. PERElson ANd P. W. Nelson, Mathematical analysis of HIV-1 dynamics in vivo, SIAM Rev., 41 (1999), pp. 3-44 (electronic).

[65] A. SAUL, Models for the in-host dynamics of malaria revisited: errors in some basic models lead to large over-estimates of growth rates., Parasitology, 117 ( Pt 5) (1998), pp. 405-407; discussion 409-410.

[66] C. P. Simon, J. A. Jacquez, And J. S. Koopman, A lyapunov function approach to computing $R 0$, in Models for infectious human diseases :their structure and relation to data, V. Isham and G. Medley, eds., Cambridge University Press, 1996, pp. 311-314.

[67] J. Swinton, The dynamics of blood-stage malaria : modelling strain specific and strain transcending immunity., in Models for infectious human diseases :their structure and relation to data, V. Isham and G. Medley, eds., Cambridge University Press, 1996, pp. 210-212.

[68] H. R. THIEME, Global asymptotic stability in epidemic models, in Equadiff 82 proceedings, W Knobloch and K Schmitt, eds., Lecture notes in Math,1017, Springer-Verlag, 1983, pp. 609615.

[69] _ Mathematics in population biology, Princeton Series in Theoretical and Computational Biology, Princeton University Press, Princeton, NJ, 2003.

[70] M. VIDYASAGAR, Decomposition techniques for large-scale systems with nonadditive interactions :stability and stabilizability, IEEE Trans. Automat. Control, AC-25 (1980), pp. 773 779.

[71] P. VAN DEN DRIESSChE AND J. WATMOugh, reproduction numbers and sub-threshold endemic equilibria for compartmental models of disease transmission. Math. Biosci., 180, 2002. 